\title{
Quantum Mechanics without Waves: a Generalization of Classical Statistical Mechanics
}

\author{
Marcello Cini \\ Dipartimento di Fisica - Università La Sapienza - Roma \\ INFM Sezione di Roma - La Sapienza
}

October 10, 2018

\begin{abstract}
We generalize classical statistical mechanics to describe the kynematics and the dynamics of systems whose variables are constrained by a single quantum postulate (discreteness of the spectrum of values of at least one variable of the theory). This is possible provided we adopt Feynman's suggestion of dropping the assumption that the probability for an event must always be a positive number. This approach has the advantage of allowing a reformulation of quantum theory in phase space without introducing the unphysical concept of probability amplitudes, together with all the problems concerning their ambiguous properties.
\end{abstract}

(1) Postal address: Piazza A.Moro, 2, 00185 Roma, Italy. E-mail: marcello.cini@roma1.infn.it. 


\section{Introduction}

After seventy years of Quantum Mechanics we have learned to live with complex probability amplitudes without worrying about their lack of any reasonable physical meaning. One should not ignore, however, that the "wavelike" properties of quantum objects still raise conceptual problems on whose solutions a general consensus is far from having been reached ${ }^{(1)(2)}$.

A possible way out of this difficulty has been implicitly suggested by Feynman ${ }^{(3)}$, who has shown that, by dropping the assumption that the probability for an event must always be a nonnegative number, one can avoid the use of probability amplitudes in quantum mechanics. This proposal, which goes back to the work of Wigner ${ }^{(4)}$ who first introduced non positive pseudoprobabilities to represent Quantum Mechanics in phase space, does not, however, eliminate "waves", because its starting point is the conventional mathematical framework of Quantum Mechanics.

We try instead to reformulate quantum mechanics by eliminating from

the beginning the concept of probability "waves". This program is carried on by generalizing the formalism of classical statistical mechanics in phase space with the introduction of a single quantum postulate (discreteness of the spectrum of values of at least one variable of the theory), which introduces mathematical constraints on the variables in terms of which any physical quantity can be expressed (characteristic variables). These constraints, however, cannot be fulfilled by ordinary random c-numbers, but are satisfied 
by q-numbers. The introduction of q-numbers in quantum theory is therefore not assumed as a postulate from the beginning, but is a consequence of a well defined physical requirement. The equations derived from these constraints allow the determination of the expectation value of the characteristic variables for any given dispersion-free ensemble together with the value of the physical quantity which defines it. This leads to the identification of the characteristic variables with the Weyl operators of standard Quantum Mechanics. The whole structure of Quantum Mechanics in phase space, including the identification of the Wigner function as the pseudoprobability density of any quantum state, derived by Moyal in his pioneer work of $1949^{(5)}$, is therefore deduced from a single quantum postulate without ever introducing wave functions or probability amplitudes.

\section{Classical statistical mechanics in phase space}

Consider a classical statistical ensemble of systems whose state may be defined by the values of a couple of conjugated variables, $\mathbf{q}, \mathbf{p}$, which can take the values $q, p$, respectively. The standard form of the joint probability density ist

\footnotetext{
${ }^{1}$ The constant $\hbar$ is introduced here as a unit of action for dimensional reasons. The classical results are independent of its value. Its identification with Planck constant will result from the comparison of quantum theory with experiment.
} 
$P(q, p)=<\delta(q-\mathbf{q}) \delta(p-\mathbf{p})>=(2 \pi \hbar)^{-2} \iint d y d k e^{(-i / \hbar)(k q+y p)}<e^{(i / \hbar)(k \mathbf{q}+y \mathbf{p})}>$

where $<$.> represents the ensemble average. Similarly, any physical quantity $A(\mathbf{q}, \mathbf{p})$ (for short $\mathbf{A})$ can be expressed in terms of the same variables $e^{(i / \hbar)(k \mathbf{q}+y \mathbf{p})}$ (hereafter indicated as characteristic variables), as

$$
\mathbf{A}=\iint d y d k a(k, y) e^{(i / \hbar)(k \mathbf{q}+y \mathbf{p})}
$$

It is useful for our later generalization to introduce the notation $\mathbf{C}(k, y)$ for the characteristic variables $e^{(i / \hbar)(k \mathbf{q}+y \mathbf{p})}$. Let us consider an ensemble in which all the systems have the same value $\alpha$ of the physical quantity $\mathbf{A}$. Then it must be

$$
<\mathbf{A}^{2}>_{\alpha}=\alpha^{2}
$$

where

$$
\alpha=<\mathbf{A}>_{\alpha}=\iint d q d p A(q, p) P_{\alpha}(q, p)
$$

In order to satisfy eqs. (3) the function $\left\langle\mathbf{C}(k, y)>_{\alpha}\right.$ of $k, y$ (indicated in the following as characteristic function of the ensemble) given by the Fourier inversion of eq. (1) must obey the relation

$$
\alpha<\mathbf{C}(k, y)>_{\alpha}=\iint d x d h a(h-k, x-y)<\mathbf{C}(h, x)>_{\alpha}
$$


for any given value $\alpha$ of $\mathbf{A}$. To derive (5) it is crucial to use the property

$$
\mathbf{C}(k, y) \mathbf{C}\left(k^{\prime}, y^{\prime}\right)=\mathbf{C}\left(k+k^{\prime}, y+y^{\prime}\right)
$$

Eq. (5) is an homogeneous integral equation for the determination of the eigenvalues $\alpha$ of $\mathbf{A}$ and the corresponding eigenfunctions $\langle\mathbf{C}(k, y)\rangle_{\alpha}$. Its solutions can be immediately obtained from its inverse Fourier transform. In terms of $A(q, p)$ (the inverse Fourier transform of $a(k, y))$ and of $P_{\alpha}(q, p)$ eq. (5) shows to be no longer an integral equation but a simple algebraic equation:

$$
A(q, p) P_{\alpha}(q, p)=\alpha P_{\alpha}(q, p)
$$

which has the solutions

$$
\begin{gathered}
\alpha=A(q, p) \\
P_{\alpha}(q, p)=f_{\alpha}(\Pi(q, p)) \quad \delta(A(q, p)-\alpha)
\end{gathered}
$$

with $f(\boldsymbol{\Pi})$ an arbitrary function of the variable $\boldsymbol{\Pi}$ conjugated to $\mathbf{A}$. In fact any other dependence of $f_{\alpha}$ on q and $\mathrm{p}$ could be expressed as a dependence on $A(q, p)$ which would be eliminated by replacing $A(q, p)$ with $\alpha$. This arbitrariness reflects the fact that there may be an infinity of classical ensembles in which the variable $\mathbf{A}$ has the value $\alpha$. Eq. (7) implies that, given a couple 
of values $\mathrm{q}, \mathrm{p}$ of the variables $\mathbf{q}, \mathbf{p}$, the variable $\mathbf{A}$ has necessarily the value (8). This seems a trivial statement but it will turn out to be essential later. It should be noted that (9) holds for any dynamical variable, function of $\mathbf{q , p}$. The case of the energy is no exception, in spite of the questionable role of the time as its conjugate variable, since the Hamiltonian $\mathbf{H}=\mathrm{H}(\mathbf{q}, \mathbf{p})$ can be expressed, by means of a suitable canonical transformation $\mathbf{Q}=\mathrm{Q}(\mathbf{q}, \mathbf{p})$, $\mathbf{P}=\mathrm{P}(\mathbf{q}, \mathbf{p})$, as a function $\mathrm{E}(\mathbf{P})$ of the new momentum not depending on the new coordinate $\mathbf{Q}$. Therefore a given value $\mathrm{P}$ of $\mathbf{P}$ yields a uniquely determined value $\mathrm{E}(\mathrm{P})$ of the energy. We can take therefore $\boldsymbol{\Pi}=\mathbf{Q}$. For closed systems $\mathbf{P}$ is the action variable $\mathbf{J}=\mathrm{J}(\mathbf{q}, \mathbf{p})$, and $\mathrm{E}(\mathrm{J})$ is independent of the conjugated angle variable $\boldsymbol{\Theta}=\mathbf{Q}$ of $\mathbf{J}$.

The limiting case $f=$ constant is the most interesting for the generalization we have in mind, because in this particular classical ensemble the variable $\mathbf{\Pi}$ is completely undetermined.

In this case and only in this case the ensemble acquires a very important property. In fact, by indicating with $\{., .\}_{P B}$ the Poisson Bracket of A with an arbitrary variable $\mathbf{B}$, one has

$$
\begin{aligned}
<\{\mathbf{A}, \mathbf{B}\}_{P B}>_{\alpha} & =-\iint d p d q P_{\alpha}(p, q)[(\partial A / \partial q)(\partial B / \partial p)-(\partial A / \partial p)(\partial B / \partial q)]= \\
& =-\iint d p d q B(p, q)\left[(\partial A / \partial q)\left(\partial P_{\alpha} / \partial p\right)-(\partial A / \partial p)\left(\partial P_{\alpha} / \partial q\right)\right]
\end{aligned}
$$

namely 


$$
<\{\mathbf{A}, \mathbf{B}\}_{P B}>_{\alpha}=0
$$

because when $P_{\alpha}$ depends only on A we have $\left(\partial P_{\alpha} / \partial \Pi\right)=0$.

Eq.(11) implies that both eqs.(3) and (11) are invariant under arbitrary infinitesimal canonical transformations

$$
\mathbf{A}^{\prime}=\mathbf{A}+\varepsilon\{\mathbf{A}, \mathbf{B}\}_{P B}
$$

From eq.(11) it follows therefore that, for the dispersion free ensemble in which $\mathbf{A}$ has the value $\alpha$ and $\boldsymbol{\Pi}$ is completely undetermined, the characteristic function satisfies, in addition to (5), also the equation

$$
\iint d x d h a(h-k, x-y)\left(1 / \hbar^{2}\right)(k x-h y)<\mathbf{C}(h, x)>_{\alpha}=0 .
$$

Eq.(13) represents a "classical uncertainty principle" expressing the condition to be fulfilled by classical ensembles having the property that when a given variable $\mathbf{A}$ has the value $\alpha$ the conjugate variable $\boldsymbol{\Pi}$ is undetermined. Conversely, if we impose that the characteristic function of an ensemble satisfies eqs.(5) and (13) we select only the ensembles in which the "uncertainty principle" is satisfied.

\section{Quantum postulate}

Our reformulation of quantum theory will be based on the assumption that eqs. (3) and (11) should hold for any variable A. This will impose automat- 
ically for all the possible ensembles the validity of the uncertainty principle. However the explicit form of these equations in terms of $\langle\mathbf{C}(h, x)\rangle_{\alpha}$ given by (5) and (13) will have to be modified, because eqs.(8) and (9) are no longer valid in quantum theory.

In addition to this first assumption, therefore, we will impose the fulfilment of an extra postulate, based on the convinction that, instead of postulating the conventional representation of physical quantities by means of operators in Hilbert space, it is more satisfactory to assume as a founding stone of quantum theory the experimental fact that physical quantities exist (e.g. angular momentum) whose possible values form a discrete set, invariant under canonical transformations, characteristic of each variable in question.

An equally compelling physical starting point for the adoption of this postulate might be the stability of matter. In fact this requirement implies the necessary existence of a minimum value $E_{0}$ below which no lower value can be assumed by the energy of an electron-nucleus bound state.

In any case we need only assume (Quantum Postulate) that at least one variable $\mathbf{L}$ exists which has finite gaps in the continuous range $\mathrm{L}(q, p)$ implied by its functional dependence $\mathrm{L}(\mathbf{q}, \mathbf{p})$ on $\mathbf{q}$ and $\mathbf{p}$ (which can both assume any value in the continuous range $-\infty,+\infty$ ) in which it cannot assume values except for one or more discrete values $\lambda_{i}$. This in fact means that, since $\mathbf{L}$ cannot have values in the range between $\lambda_{i}$ and $\lambda_{i}-\varepsilon$, and/or between $\lambda_{i}$ and $\lambda_{i}+\eta$, (with $\varepsilon, \eta$, finite) eqs.(8) (9) do not hold in these ranges. 
As a consequence we conclude that $\mathrm{L}(\mathbf{q}, \mathbf{p})$ cannot be expressed in the form (2), namely that the quantum characteristic variables $\mathbf{C}(\mathrm{k}, \mathrm{y})$ cannot satisfy the crucial property (6).

Therefore, since by definition all variables should be expressed in terms of a unique set of characteristic variables, we conclude that for all the variablest $\hat{\mathbf{A}}$, eq.(2) should be replaced by

$$
\hat{\mathbf{A}}=\iint d y d k a(k, y) \hat{\mathbf{C}}(k, y)
$$

with a set of characteristic variables $\hat{\mathbf{C}}(k, y)$ obeying a new rule of multiplication replacing eq.(6).

In order to find the required modification of eq.(6) we start by asking how the eigenvalue equation (5) should be modified in order to allow, besides (instead or in addition to) a continuous range of possible values, also for the existence of discrete eigenvalues $\lambda_{i}$ of $\hat{\mathbf{L}}$. This amounts to say that its Fourier transform should no longer reduce to the algebraic relation (7) but should become a true Fredholm homogeneous integral equation, which, as is well known, has the property, under suitable conditions, of allowing for the existence of discrete eigenvalues.

Whether a given variable $\hat{\mathbf{A}}$ will actually have eigenvalues belonging to a discrete or a continuous (or even both) spectrum will depend on its functional dependence on $\hat{\mathbf{p}}$ and $\hat{\mathbf{q}}$. There are in any case some stringent requirements

\footnotetext{
${ }^{2}$ We change the notation from $\mathbf{A}$ to $\hat{\mathbf{A}}$ in order to distinguish the variables satisfying the Quantum Postulate from those satisfying (2)
} 
that the modified kernel should satisfy to attain this goal, namely:

a) the basic information on the functional dependence of $\hat{\mathbf{A}}$ on $\hat{\mathbf{p}}$ and $\hat{\mathbf{q}}$ contained in the kernel a(h-k, x-y) should remain unaltered;

b) the correlation between the couple of variables $h, x$ and $k, y$ which is necessary in order to transform eq.(5) into a true Fredholm integral equation should be universal, namely independent of the variable chosen and of the state considered;

c) the classical kernel should be recovered when $k=y=0$ because eq. (5) for $<\hat{\mathbf{C}}(0,0)>_{\alpha}=1$ should give eq.(4) which must still be valid;

d) the classical kernel should be recovered also for $h=x=0$ because the relation (6) should still be valid when $k=h$ and $x=y$.

The simplest (and from this point of view unique) way to satisfy a) and b) is to multiply the classical kernel $a(h-k, x-y)$ by a factor $g(k x-h y)$ whose argument is unambiguously fixed by the requirement that, for dimensional reasons, $x$ should be correlated to $k$ and $h$ to $y$. Furthermore in order that c) and d) are fulfilled, it must be $\mathrm{g}(0)=1$. The modified integral equation replacing eq. (5) should therefore read

$$
\alpha_{i}<\hat{\mathbf{C}}(k, y)>_{i}=\iint d x d h a(h-k, x-y) \mathrm{g}(k x-h y)<\hat{\mathbf{C}}(h, x)>_{i}
$$

Eq. (15) has a first important consequence. In fact the condition (3), which may be rewritten in terms of the new variables $\hat{\mathbf{C}}(k, y)$ in the form 


$$
\begin{aligned}
& \iint d y d k a(k, y) \iint d y^{\prime} d k^{\prime} a\left(k^{\prime}, y^{\prime}\right)<\hat{\mathbf{C}}(k, y) \hat{\mathbf{C}}\left(k^{\prime}, y^{\prime}\right)>_{i} \\
& =\alpha_{i} \iint d y d k a(k, y)<\hat{\mathbf{C}}(k, y)>_{i}
\end{aligned}
$$

leads to eq. (15) only if eq. (6) is replaced by

$$
(1 / 2)\left[\hat{\mathbf{C}}(k, y) \hat{\mathbf{C}}\left(k^{\prime}, y^{\prime}\right)+\hat{\mathbf{C}}\left(k^{\prime}, y^{\prime}\right) \hat{\mathbf{C}}(k, y)\right]=g\left(k y^{\prime}-k^{\prime} y\right) \hat{\mathbf{C}}\left[\left(k+k^{\prime}\right),\left(y+y^{\prime}\right)\right]
$$

This equation, however, cannot be satisfied by ordinary c-numbers. This means that, if we want to allow for the existence of discrete values of at least one variable $\hat{\mathbf{L}}$ we are forced to represent all the variables $\hat{\mathbf{A}}$ by means of q-numbers. We need not, however assume for these q-number variables other properties except that they exist and that (17) is satisfied. This means that the mathematical nature of the entities needed to represent the quantum variables is a consequence of the physical property represented by our Quantum Postulate, and not viceversa, as the conventional view of reality underlying the conventional axiomatic formulation of Quantum Mecchanics assumes.

We need not give any new rule in order to define the symbol $<\hat{\mathbf{C}}(k, x)>_{i}$ in terms of operators and state vectors, because on the one hand its physical meaning is by definition the same of its classical counterpart $\left\langle e^{(i / \hbar)(k \mathbf{q}+y \mathbf{p})}\right\rangle_{i}$ namely the mean value of the characteristic variable in the ensemble in which the variable $\hat{\mathbf{A}}$ has the value $\alpha_{i}$, and on the other hand its explicit expression will be derived by solving eq. (15) together with the analogous quantum generalization of eq. (13) which we will now proceed to write down. 
In order to fulfill the condition that the eigenvalues $\alpha_{i}$ of $\hat{\mathbf{A}}$ should be invariant under the canonical transformations (12) one must in fact impose that eq. (11) should hold. However, if we use for the new characteristic variables $\hat{\mathbf{C}}(k, y)$ the classical PBs of the old variables

$$
\left\{e^{(i / \hbar)(k \mathbf{q}+y \mathbf{p})}, e^{(i / \hbar)\left(k^{\prime} \mathbf{q}+y^{\prime} \mathbf{p}\right)}\right\}_{P B}=\left[\left(k^{\prime} y-k y^{\prime}\right) / \hbar^{2}\right] e^{(i / \hbar)\left[\left(k+k^{\prime}\right) \mathbf{q}+\left(y+y^{\prime}\right) \mathbf{p}\right]}
$$

we immediately see that eq. (17) is no longer invariant under (12) which has therefore to be replaced by

$$
\hat{\mathbf{A}}^{\prime}=\hat{\mathbf{A}}+\varepsilon\{\hat{\mathbf{A}}, \hat{\mathbf{B}}\}_{Q P B}
$$

We have therefore to derive the corresponding quantum Poisson Brackets (QPB) of the two variables $\hat{\mathbf{C}}(k, y), \hat{\mathbf{C}}\left(k^{\prime}, y^{\prime}\right)$ from the condition of invariance of (17) under (19). Here again we need not introduce explicitly the standard definition of the PB's of these q-numbers in terms of operators. On the contrary, their form will be obtained as a consequence of our formalism. We will only need to define QPB's, for consistency with eq. (17), by means of the following generalization of the classical PBs

$$
\left\{\hat{\mathbf{C}}(k, y), \hat{\mathbf{C}}\left(k^{\prime}, y^{\prime}\right)\right\}_{Q P B}=\mathrm{f}\left(k y^{\prime}-k^{\prime} y\right) \hat{\mathbf{C}}\left[\left(k+k^{\prime}\right),\left(y+y^{\prime}\right)\right]
$$

where $f(\lambda)$ is an odd function of its argument satisfying, for consistency with eq. (18), the condition $\lim _{\lambda \rightarrow 0} f(\lambda)=-\lambda / \hbar^{2}$

From (17) and (20) we now obtain immediately

$$
\iint d x d h a(h-k, x-y) \mathrm{f}(k x-h y)<\hat{\mathbf{C}}(h, x)>_{i}=0
$$


This is the required generalization of eq. (13).

The further step required to complete our formalism is the determination of the functions $f($.$) and g($.$) . The knowledge of these functions will then al-$ low the explicit derivation of the characteristic function $<\hat{\mathbf{C}}(k, y)>_{i}$ and the corresponding eigenvalue $\alpha_{i}$ of $\hat{\mathbf{A}}$ for any state $<>_{\mathrm{i}}$ by solving eqs. (15) and (21). This goal is easily attained by imposing the condition that both relations (16) and (20) should be invariant under the canonical transformations (19). This condition leads in fact to the two equations

$$
\begin{gathered}
\mathrm{f}(\lambda) \mathrm{f}(\mu-\nu)+\mathrm{f}(\mu) \mathrm{f}(\nu-\lambda)+\mathrm{f}(\nu) \mathrm{f}(\lambda-\mu)=0 \quad(\text { Jacobi identity }) \\
\mathrm{g}(\lambda) \mathrm{f}(\mu+\nu)=\mathrm{g}(\lambda+\mu) \mathrm{f}(\nu)+\mathrm{g}(\lambda-\nu) \mathrm{f}(\mu)
\end{gathered}
$$

These equations have the following solutions

$\mathrm{g}\left(k y^{\prime}-k^{\prime} y\right)=\cos \left[b\left(k y^{\prime}-k^{\prime} y\right) / \hbar\right] ; \quad \mathrm{f}\left(k y^{\prime}-k^{\prime} y\right)=(1 / b \hbar) \sin \left[b\left(k^{\prime} y-k y^{\prime}\right) / \hbar\right]$

with $b$ a parameter which is still undetermined. It should be stressed that the classical statistical theory is not recuperated by making $\hbar \rightarrow 0$, but by letting the adimensional parameter $b \rightarrow 0$ (absence of correlations). However, although $b \rightarrow 0$ is a valid mathematical limit for the expressions (25), $b=0$ and $b \neq 0$ yield two radically different theories, because in the first case the variables are c-numbers while in the second one they are q-numbers. 
The solution $<\hat{\mathbf{C}}(k, y)>_{i}$ of eqs. (15) (21) will now yield easily the corresponding expression for $P_{i}(q, p)$ by means of

$$
P_{i}(q, p)=(2 \pi \hbar)^{-2} \iint d y d k e^{(-i / \hbar)(k q+y p)}<\mathbf{C}(k, y)>_{i}
$$

Before discussing the properties of this (pseudo)probability density we will however work out the results of our theory in some simple cases.

\section{Simple examples}

We will first solve the two equations (15) (21) for the variables $\hat{\mathbf{q}}, \hat{\mathbf{p}}$ and suc-

cessively for the energy $\hat{\mathbf{H}}=(1 / 2) \hat{\mathbf{p}}^{2}+(1 / 2) \omega^{2} \hat{\mathbf{q}}^{2}$ of the harmonic oscillator. This will show explicitly how the formalism leads both to the existence of variables whose eigenvalues belong to a continuous range as well as of other ones with a discrete spectrum.

1. Variable $\hat{\mathbf{q}}$. From the classical expression (2) one obtains

$$
a_{q}(k, y)=\iint d q d p q \exp [-i(p y+q k) / \hbar]=i \hbar \delta(y)[\partial \delta(k) / \partial k]
$$

The eigenvalue equation (15) reads

$$
\begin{aligned}
q_{o}<\hat{\mathbf{C}}(k, y)>_{q_{o}} & =i \hbar \iint d x d h \delta(x-y)[\partial \delta(h-k) / \partial h] \mathrm{g}(k x-h y)<\hat{\mathbf{C}}(k, y)>_{q_{o}}= \\
& =-i \hbar\left[\partial<\hat{\mathbf{C}}(k, y)>_{q_{o}} / \partial k\right]
\end{aligned}
$$

because $\mathrm{g}(0)=1$ and $[\partial \mathrm{g}(\lambda) / \partial \lambda]_{\lambda=0}=0$, where $q_{0}$ is the value of $\hat{\mathbf{q}}$ which labels the state. The solution of (27) is

$$
<\hat{\mathbf{C}}(k, y)>_{q_{o}}=\exp \left[i k q_{o} / \hbar\right] \phi(y)
$$


with $\phi(y)$ an arbitrary function. On the other hand eq. (21) reads

$0=i \hbar \iint d x d h \delta(x-y)[\partial \delta(h-k) / \partial h] f(k x-h y)<\hat{\mathbf{C}}(h, x)>_{q_{o}}=y<\hat{\mathbf{C}}(k, y)>_{q_{o}}$

because $\mathrm{f}(0)=0$ and $[\partial f(\lambda) / \partial \lambda]_{\lambda=0}=1 / \hbar^{2}$. Eq. (29) gives immediately

$$
\phi(y)=\delta(y)
$$

By introducing (28) (30) in eq. (25) one obtains

$$
P_{q_{o}}(q, p)=(2 \pi \hbar)^{-1} \delta\left(q-q_{o}\right)
$$

which coincides with the classical probability density of the ensemble in which the variable $\hat{\mathbf{q}}$ has the value $q_{o}$.

This shows that the possible values of the quantum variable $\hat{\mathbf{q}}$ span the same continuous range from $-\infty$ to $+\infty$ of the classical variable $\hat{\mathbf{q}}$. This is because the solution of (27) and (29) involves only the classical limits of $\mathrm{g}($. and $\mathrm{f}($.$) and does not depend on the actual value of b$. In this case the QPB's coincide with the classical PB's.

2. Variable $\hat{\mathbf{p}}$. The complete symmetry between $\hat{\mathbf{q}}$ and $\hat{\mathbf{q}}$ allows us to write

$$
<\hat{\mathbf{C}}(k, y)>_{p_{o}}=\exp \left[i y p_{o} / \hbar\right] \delta(k)
$$

namely

$$
P_{p_{o}}(q, p)=(2 \pi \hbar)^{-1} \delta\left(p-p_{o}\right)
$$


3. Variable $\hat{\mathbf{H}}=(1 / 2) \hat{\mathbf{p}}^{2}+(1 / 2) \omega^{2} \hat{\mathbf{q}}^{2}$. From the classical expression we obtain

$$
\begin{aligned}
h(k, y) & =\iint d q d p(1 / 2)\left[p^{2}+\omega^{2} q^{2}\right] \exp [-i(p y+q k) / \hbar]= \\
& =-\left(\hbar^{2} / 2\right)\left[\delta(k) \partial^{2} \delta(y) / \partial y^{2}+\omega^{2} \delta(y) \partial^{2} \delta(k) / \partial k^{2}\right]
\end{aligned}
$$

Eq. (15) reads

$$
\begin{aligned}
& E_{o}<\hat{\mathbf{C}}(k, y)>_{E_{o}}= \\
& =-\left(\hbar^{2} / 2\right) \iint d x d h \delta(x-y) \delta(h-k)\left[\partial^{2} / \partial x^{2}+\omega^{2} \partial^{2} / \partial h^{2}\right]\left[g(k x-h y)<\hat{\mathbf{C}}(h, x)>_{E_{o}}\right]
\end{aligned}
$$

Since, from (24) we have

$$
\begin{aligned}
& {\left[\partial^{2} \mathrm{~g}(h y-k x) / \partial x^{2}\right]_{x=y ; h=k}=-k^{2} b^{2} / \hbar^{2}} \\
& {\left[\partial^{2} \mathrm{~g}(h y-k x) / \partial h^{2}\right]_{x=y ; h=k}=-y^{2} b^{2} / \hbar^{2}}
\end{aligned}
$$

we obtain

$$
E_{o}<\hat{\mathbf{C}}(k, y)>_{E_{o}}=(1 / 2)\left[k^{2} b^{2}-\hbar^{2} \omega^{2} \partial^{2} / \partial k^{2}+\omega^{2} y^{2} b^{2}-\hbar^{2} \partial^{2} / \partial k^{2}\right]<\hat{\mathbf{C}}(k, y)>_{E_{o}}
$$

From eq. (21) we obtain

$$
\left[k \partial / \partial y-\omega^{2} y \partial / \partial k\right]<\hat{\mathbf{C}}(k, y)>_{E_{o}}=0
$$

Eq. (38) can be solved by setting

$$
<\hat{\mathbf{C}}(k, y)>_{E_{o}}=F(k) G(y)
$$

leading to

$$
F(k)=\exp \left[\mu k^{2} / \omega^{2}\right] \quad G(y)=\exp \left[\mu y^{2}\right]
$$


Introducing (39) and (40) in (37) we easily find (since $<\hat{\mathbf{C}}(0,0)>=1$ )

$$
\begin{gathered}
E_{o}=b \hbar \omega \\
<\hat{\mathbf{C}}(k, y)>_{E_{o}}=\exp \left[-b k^{2} / 2 \hbar \omega\right] \exp \left[-b y^{2} \omega / 2 \hbar\right]
\end{gathered}
$$

By introducing (42) in (25) we obtain

$$
P_{E_{o}}(q, p)=(1 / 2 \pi \hbar b) \exp \left[-p^{2} / 2 \hbar \omega b\right] \exp \left[-q^{2} \omega / 2 \hbar b\right]
$$

For the excited states the separability condition (39) does not hold. Eqs. (37) and(38) are however sufficient to determine completely the corresponding characteristic functions and eigenvalues ${ }^{(10)}$.

\section{The uncertainty principle}

We will finally discuss the properties of the (pseudo)probability densities $P_{i}(q, p)$ given by $(25)$. This will also allow us to determine the parameter $b$.

We start by writing eq. (21) for both $<\hat{\mathbf{C}}(k, y)>_{i}$ and $<\hat{\mathbf{C}}(k, y)>_{j}$ for $i \neq j$; we multiply the first one by $<\hat{\mathbf{C}}(-k,-y)>_{j}$ and the second one by $<\hat{\mathbf{C}}(-k,-y)>_{i}$ and finally integrate over $k, y$. By subtracting the second equation from the first one we obtain

$$
0=\left(\alpha_{i}-\alpha_{j}\right) \iint d y d k<\hat{\mathbf{C}}(-k,-y)>_{i}<\hat{\mathbf{C}}(k, y)>_{j}
$$


This amounts to writing]

$$
\iint d y d k<\hat{\mathbf{C}}(-k,-y)>_{i}<\hat{\mathbf{C}}(k, y)>_{j}=N \delta_{i j}
$$

where $N$ is a normalization constant, having the dimensions of an action, independent of the variable $\hat{\mathbf{A}}$ and of the state $<>_{i}$. From (25) and (45) we obtain

$$
\iint d q d p\left[(2 \pi \hbar)^{2} P_{i}(q, p) / N\right] P_{j}(q, p)=\delta_{i j}
$$

At this stage we have to fix our unit of action $2 \pi \hbar$. To this purpose we compare (46) with its semiclassical limit given by the old theory of quanta of Planck and Bohr where the volume of the region of phase space in which the classical $A(q, p)$ has the value $\alpha_{i}$ and all the points $q, p$ have equal constant probability $K_{i}$ inside it and zero probability outside, is assumed to be equal to Planck's constant $(2 \pi \hbar)$. Then in this semiclassical theory, we have, for the normalization of probability

$$
2 \pi \hbar K_{i}=1
$$

and for (46)

$$
(2 \pi \hbar)^{3} K_{i}^{2} / N=1
$$

from which we get $N=2 \pi \hbar$. Eq. (46) becomes therefore

$$
\iint d q d p P_{i}(q, p) P_{i}(q, p)=(2 \pi \hbar)^{-1} \equiv P_{a v}
$$

\footnotetext{
${ }^{3}$ We assume that the spectrum is nondegenerate.
} 
The last step of our work is now the determination of the parameter $b$. In fact it is immediate to see that, introducing into eq. (50) the expression (43) for $P_{E_{o}}(q, p)$ of the harmonic oscillator ground state, one obtains $b=$ $1 / 2$. Since this value is independent of the variable and of the state chosen, this result is wholly general and consequently our reformulation of quantum theory is complete.

Eq. (50) expresses a new form of the uncertainty principle for position and momentum. In fact, by introducing in the normalization condition the mean value $P_{a v}$ defined by this equation, we obtain

$$
\iint d q d p P(q, p)=P_{a v} \delta q \delta p=1
$$

where $\delta q \delta p$ is the volume of phase space in which $P(q, p)$ is replaced by $P_{a v}$ and is zero outside. We then immediately obtain

$$
\delta q \delta p=2 \pi \hbar .
$$

It is important to stress that (52) does not have the form of the conventional Heisenberg inequality, which gives no upper limit to the possible value of the uncertainty product $\Delta q \Delta p$ of the mean square values of $q$ and $p$, but involves only its minimum value. We will return on the implications of this difference in the discussion. 


\section{The dynamical evolution}

We finally indicate how the dynamical evolution of the pseudoprobability distribution $P(q, p)$ in any given state given by $(25)$ can be worked out. It is sufficient to use the Hamiltonian $\hat{\mathbf{H}}$

$$
\hat{\mathbf{H}}=\iint d y d k h(k, y) \hat{\mathbf{C}}(k, y)
$$

as the generator of the infinitesimal displacement in time

$$
d \hat{\mathbf{C}}(k, y) / d t=\{\hat{\mathbf{C}}(k, y), \hat{\mathbf{H}}\}_{Q P B}=\iint d x d j h(j-k, z-y) f(j y-k x) \hat{\mathbf{C}}(j, x)
$$

Eq. (54) yields a Chapman-Kolmogorov equation for the time dependence of the pseudoprobability density $P(q, p ; t)$ :

$$
(d / d t) P(q, p ; t)=\iint d q^{\prime} d p^{\prime} K\left(q, p ; q^{\prime}, p^{\prime}\right) P\left(q^{\prime}, p^{\prime} ; t\right)
$$

In the classical limit eq.(55) reduces to the Liouville equation.

We have therefore attained our goal, namely the construction of a formal probabilistic theory (with the generalization of probabilities to negative values according to Feynman's interpretation) of the quantum world in phase space by means of a straightforward generalization of classical statistical mechanics.

\section{Comparison with the conventional formu- lation of Quantum Mechanics}

The present formulation of quantum theory is clearly identical to the conventional formalism of Quantum Mechanics. In fact if we consider the Weyl 
operator

$$
\underline{\mathbf{C}}(k, y)=e^{i(\underline{\mathbf{p}} y+k \underline{\mathbf{q}}) / \hbar}
$$

where $\underline{\mathbf{p}}$ and $\underline{\mathbf{q}}$ are the momentum and position operators satisfying the usual commutation relation $[\underline{\mathbf{q}}, \underline{\mathbf{p}}]=i \hbar$, one finds immediately that

$$
\hat{\mathbf{C}}(k, y)=\underline{\mathbf{C}}(k, y)
$$

Therefore, if $|\psi\rangle$ is the state vector corresponding to our state $\langle$. $>$ we have

$$
\begin{gathered}
<\hat{\mathbf{C}}(k, y) \hat{\mathbf{C}}\left(k^{\prime}, y^{\prime}\right)+\hat{\mathbf{C}}\left(k^{\prime}, y^{\prime}\right) \hat{\mathbf{C}}(k, y)>=2 R e<\psi\left|\underline{\mathbf{C}}(k, y) \underline{\mathbf{C}}\left(k^{\prime}, y^{\prime}\right)\right| \psi> \\
<\left\{\hat{\mathbf{C}}(k, y), \hat{\mathbf{C}}\left(k^{\prime}, y^{\prime}\right)\right\}_{Q P B}>=(2 / \hbar) \operatorname{Im}<\psi\left|\underline{\mathbf{C}}(k, y) \underline{\mathbf{C}}\left(k^{\prime}, y^{\prime}\right)\right| \psi>
\end{gathered}
$$

From (57) (58) it follows also that

$$
P(q, p)=W(q, p)
$$

where $W(q, p)$ is the Wigner function ${ }^{(4)}$ of the state $\mid \psi>$. Eq. (55) coincides therefore with the standard equation for the time evolution of the Wigner function. This result shows that this function has a privileged status among other functions ${ }^{(6)}$ used in the literature to describe Quantum Mechanics in phase space, because it can be derived directly from our quantum postulate.

\section{Discussion}

The physical meaning of negative probabilities is well clarified by Feynman's own words: "It is that a situation for which a negative probability is calculated 
is impossible, not in the sense that the chance for its happening is zero, but rather in the sense that the assumed conditions of preparation or verification are experimentally unattainable." Admittedly, as he recognizes, a "strong mental block" against this extention of the probability concept is widespread. Once this has been overcome, however, the present formulation of quantum theory has several advantages.

First of all, as already anticipated in the introduction, many paradoxes typical of the wave-particle duality disappear. On the one hand in fact, as already shown by Feynman, it becomes possible to express the correlations between two distant particles in terms of the product of two probabilities independent from each other ${ }^{(3)(7)}$. All the speculations on the nature of an hypothetical superluminal signal between them becomes therefore meaningless. On the other hand the long time debated question about the meaning of the superposition of state vectors for macroscopic objects (Schrödinger's cats) may also be set aside as equally baseless, together with the many proposals of detection of "empty waves". It is not the practical use of the formalism of Quantum Mecanics, of course, which is put in question. However, from a conceptual point of view, the elimination of the waves from quantum theory is in line with the procedure inaugurated by Einstein with the elimination of the aether in the theory of electromagnetism.

Secondly, this approach eliminates the conventional hybrid procedure of describing the dynamical evolution of a system, which consists of a first stage 
in which the theory provides a deterministic evolution of the wave function, followed by a hand made construction of the physically meaningful probability distributions. If the probabilistic nature of the microscopic phenomena is fundamental, and not simply due to our ignorance as in classical statistical mechanics, why should it be impossible to describe them in probabilistic terms from the very beginning?

The third advantage is connected with the possibility of dissipating the ambiguity of the conventional theory about two physically different aspects of the quantum uncertainties inherent to the Heisenberg inequality. It has been recognized in fact that this inequality contains two contributions of different origin $^{(8)}$. Its minimum value is in fact an ontological uncertainty, of quantum nature, while the contribution exceeding this minimum is of epistemic nature, namely expresses a statistical effect due to imperfect knowledge of reality. In fact, while the irreducible quantum contribution requires that a reduction of $\Delta x$ should necessarily imply a simultaneous increase of $\Delta p$ (or viceversa), for the statistical contribution both uncertainties can be reduced at the same time by more accurate measurements until the minimum value is reached. In the present formulation of quantum theory, however, only the quantum ontological uncertainties are present, without any spurious statistical contribution. This is because the uncertainty principle in our theory is given by the equality (52), involving only the minimum value of the Heisenberg inequality. 
The last, but not least, appeal of this approach is that it may be cosidered as a conceptual "Gestalt switch" of the type suggested by Thomas Kuhn ${ }^{(9)}$ concerning the status of the "Laws of Nature". A switch from the "autocratic" rule that the Laws prescribe everything that must happen to the "democratic" principle that anything which is not forbidden by the Laws may happen. If chance has an irreducible origin the fundamental laws cannot prescribe everything: they can only express constraints following from stability requirements of matter, or prohibitions deriving from symmetry properties of the Universe, or general principles warranting the existence of patterns of order. In other words they should allow for the occurrence of different events under equal conditions. If this is true, it becomes meaningless to ask: how can this event happen? The answer can only be: it happens because it is not forbidden. The language of probability, suitably adapted to take into account all the relevant constraints, seems therefore to be the only language capable of expressing this fundamental role of chance.

\section{Acknowledgement}

The contribution of my friend and colleague Gianni Jona-Lasinio has been crucial in clarifying the mathematical nature of the characteristic variables and in giving to the formulation of the theory a tight logical structure. I wish therefore to express my gratitude for his constructive criticism, without which this paper would not have been accomplished. Illuminating discussions 
with Francesco Guerra and Maurizio Serva are also gratefully acknowledged. 


\section{References}

[1] A. Einstein, B. Podolsky, N. Rosen, Phys. Rev. 47, 777 (1935); J.S. Bell, Physics, 1, 195 (1964).

[2] E. Schrödinger, Naturwissenshaften, 49, 53 (1935); M. Cini, Nuovo Cim. 73B, 27 (1983).

[3] R. Feynman, in: Quantum Implications (B.J. Hiley and F.D. Peats ed.), Routledge \& Kegan, London 1987, pag. 235.

[4] E.P. Wigner, Phys.Rev. 40, 749 (1932).

[5] J.E. Moyal, Proc. Camb. Phil. Soc. 45 (1949) 99.

[6] K.E. Cahill and R.J. Glauber, Phys. Rev. 177, 1857 (1969); 177, $1882,(1969)$.

[7] M.O. Scully, H. Walther and W. Schleich, Phys.Rev. A, 49, 182 (1994).

[8] M. Cini and M. Serva, Found. of Phys. Lett.3,129 (1990); M. Cini and M. Serva, Physics Letters A 167, 319, (1992).

[9] T.S. Kuhn - The structure of Scientific Revolutions,

[10] M. Hillery, R.F. OConnell, M.O. Scully. E.P. Wigner, Phys. Reports, $106,121,(1984)$ 